\title{
Middle East Respiratory Syndrome Coronavirus nsp1 Inhibits Host Gene Expression by Selectively Targeting mRNAs Transcribed in the Nucleus while Sparing mRNAs of Cytoplasmic Origin
}

\author{
Kumari G. Lokugamage, ${ }^{a}$ Krishna Narayanan, ${ }^{a}$ Keisuke Nakagawa, ${ }^{a}$ Kaori Terasaki, ${ }^{a}$ Sydney I. Ramirez, ${ }^{\text {b }}$ Chien-Te K. Tseng, ${ }^{\text {a,c,d,e,f }}$ \\ Shinji Makino ${ }^{\mathrm{a}, \mathrm{c}, \mathrm{d}, \mathbf{e}, \mathbf{f}}$ \\ Department of Microbiology and Immunology, ${ }^{a}$ Department of Pathology, ${ }^{b}$ Center for Biodefense and Emerging Infectious Diseases, ${ }^{\mathrm{c}}$ UTMB Center for Tropical \\ Diseases, ${ }^{d}$ Sealy Center for Vaccine Development, ${ }^{e}$ and Institute for Human Infections and Immunity, ${ }^{\mathrm{f}}$ The University of Texas Medical Branch, Galveston, Texas, USA
}

\begin{abstract}
The newly emerged Middle East respiratory syndrome coronavirus (MERS-CoV) and severe acute respiratory syndrome CoV (SARS-CoV) represent highly pathogenic human CoVs that share a property to inhibit host gene expression at the posttranscriptional level. Similar to the nonstructural protein 1 (nsp1) of SARS-CoV that inhibits host gene expression at the translational level, we report that MERS-CoV nsp1 also exhibits a conserved function to negatively regulate host gene expression by inhibiting host mRNA translation and inducing the degradation of host mRNAs. Furthermore, like SARS-CoV nsp1, the mRNA degradation activity of MERS-CoV nsp1, most probably triggered by its ability to induce an endonucleolytic RNA cleavage, was separable from its translation inhibitory function. Despite these functional similarities, MERS-CoV nsp1 used a strikingly different strategy that selectively targeted translationally competent host mRNAs for inhibition. While SARS-CoV nsp1 is localized exclusively in the cytoplasm and binds to the $40 \mathrm{~S}$ ribosomal subunit to gain access to translating mRNAs, MERS-CoV nsp1 was distributed in both the nucleus and the cytoplasm and did not bind stably to the $40 \mathrm{~S}$ subunit, suggesting a distinctly different mode of targeting translating mRNAs. Interestingly, consistent with this notion, MERS-CoV nsp1 selectively targeted mRNAs, which are transcribed in the nucleus and transported to the cytoplasm, for translation inhibition and mRNA degradation but spared exogenous mRNAs introduced directly into the cytoplasm or virus-like mRNAs that originate in the cytoplasm. Collectively, these data point toward a novel viral strategy wherein the cytoplasmic origin of MERS-CoV mRNAs facilitates their escape from the inhibitory effects of MERS-CoV nsp1.
\end{abstract}

\section{IMPORTANCE}

Middle East respiratory syndrome coronavirus (MERS-CoV) is a highly pathogenic human CoV that emerged in Saudi Arabia in 2012. MERS-CoV has a zoonotic origin and poses a major threat to public health. However, little is known about the viral factors contributing to the high virulence of MERS-CoV. Many animal viruses, including CoVs, encode proteins that interfere with host gene expression, including those involved in antiviral immune responses, and these viral proteins are often major virulence factors. The nonstructural protein 1 (nsp1) of CoVs is one such protein that inhibits host gene expression and is a major virulence factor. This study presents evidence for a strategy used by MERS-CoV nsp1 to inhibit host gene expression that has not been described previously for any viral protein. The present study represents a meaningful step toward a better understanding of the factors and molecular mechanisms governing the virulence and pathogenesis of MERS-CoV.

\footnotetext{
- oronaviruses (CoVs) carry a single-stranded, positive-sense RNA genome of $\sim 30 \mathrm{~kb}$ and are classified into four genera: alpha, beta, gamma, and delta. The Middle East respiratory syndrome (MERS) CoV (MERS-CoV), a beta CoV, emerged in Saudi Arabia in 2012 (1) and has spread to several other countries in the Middle East, North Africa, Europe, and Asia. MERS-CoV appears to have originated in bats (2), while accumulating evidence has also pointed to the dromedary camels as the potential animal reservoir $(3,4)$. MERS-CoV infection generally causes fever, cough, and pneumonia, leading to respiratory failure, and the reported case fatality rate is $\sim 40 \%$. Some MERS patients develop acute renal failure. MERS-CoV can be transmitted from person to person (5-7), and many cases have occurred in persons with chronic underlying medical conditions or immunosuppression (8). The mechanisms governing the virulence and pathogenesis of MERSCoV are largely unknown (9).

Upon entry into host cells, CoV genome expression is initiated by the translation of two large precursor polyproteins, ppla and
}

pplab, which are processed by viral proteinases into 15 to $16 \mathrm{ma}-$ ture proteins; the alpha and beta CoVs encodes 16 mature nonstructural proteins (nsp1 to nsp16), while the gamma and delta CoVs lack nsp1, the most N-terminal cleavage product, and encode only 15 nsp's (10-12). While many of these proteins play an

Received 26 May 2015 Accepted 15 August 2015

Accepted manuscript posted online 26 August 2015

Citation Lokugamage KG, Narayanan K, Nakagawa K, Terasaki K, Ramirez SI, Tseng

CK, Makino S. 2015. Middle East respiratory syndrome coronavirus nsp1 inhibits host gene expression by selectively targeting mRNAs transcribed in the nucleus while sparing mRNAs of cytoplasmic origin. J Virol 89:10970-10981. doi:10.1128/JVI.01352-15.

Editor: S. Perlman

Address correspondence to Shinji Makino, shmakino@utmb.edu.

K.G.L., K.N., and K.N. contributed equally to this article.

Copyright @ 2015, American Society for Microbiology. All Rights Reserved. 
essential role in viral RNA replication and transcription, some have other biological functions as well (12). The nsp1 proteins of alpha and beta CoVs share a biological function to inhibit host gene expression but use different strategies to exert this function (13-18). For example, nsp 1 of severe acute respiratory syndrome $\mathrm{CoV}$ (SARS-CoV), a beta $\mathrm{CoV}$, uses a two-pronged strategy to inhibit host gene expression (14); through its stable association with the 40S ribosomal subunit, it inhibits protein synthesis by inactivating the translational function of the 40 S subunit (19) and also induces host mRNA degradation by triggering an endonucleolytic RNA cleavage through the possible recruitment of a host endonuclease $(15,20)$ that results in the subsequent digestion of the cleavage mRNAs by the host exonuclease, Xrn1 (21). In contrast to SARS-CoV nsp1, nsp1 of transmissible gastroenteritis virus (TGEV), an alpha CoV, inhibits host protein synthesis without binding to the $40 \mathrm{~S}$ subunit or inducing host mRNA degradation (16). Since past studies have shown that viral proteins that inhibit host gene expression are major virulence factors $(22,23)$, the nsp 1 proteins of different CoVs, with their conserved function to inhibit host gene expression, most probably play a critical role in the pathogenesis of $\mathrm{CoV}$ infections; consistent with this notion, mouse hepatitis virus nsp1 is indeed a major virulence factor (17, 24). Hence, clarifying the molecular mechanisms by which the nsp1 of different CoVs inhibit host gene expression would contribute toward a better understanding of $\mathrm{CoV}$ virulence and pathogenesis.

In this study, we report that, like other CoV nsp1, MERS-CoV nsp1 also exhibits a conserved function to inhibit host gene expression. A comparative analysis of SARS-CoV nsp1 and MERSCoV nsp1 revealed functional similarities but mechanistic divergence among the nsp1 of these two highly pathogenic human CoVs. Our data imply that MERS-CoV nspl inhibits host gene expression by using a distinctly different strategy that has not been described previously for any viral protein. We present evidence which suggests that MERS-CoV nsp1 selectively targets the nuclear-transcribed endogenous host mRNAs for inhibition, whereas mRNAs that are cytoplasmic in origin, including MERS-CoV mRNAs, escape the inhibitory effects of MERS-CoV nsp1. We propose this property of MERS-CoV nsp 1 to distinguish between cellular and viral mRNAs as a novel viral escape strategy that downregulates the expression of host antiviral proteins while facilitating the expression of viral proteins in MERS-CoV-infected cells.

\section{MATERIALS AND METHODS}

Cells and virus. Vero E6 cells and BSR-T7/5 cells were grown in minimum essential medium supplemented with $10 \%$ fetal bovine serum, and 293 cells were maintained in Dulbecco modified Eagle medium supplemented with $10 \%$ fetal bovine serum. The EMC/2012 strain of MERS-CoV (25) was grown and titrated on Vero E6 cells.

Plasmid construction. Human-codon optimized synthetic DNA encoding MERS-CoV nsp1 carrying a C-terminal myc tag was cloned into pCAGGS-MCS, resulting in pCAGGS-MERS-CoV-nsp1. Insertion of the DNA fragment encoding the codon-optimized MERS-CoV nsp1 into pcDNA-MCS yielded pcDNA-MERS-nspl. The constructs, pCAGGSMERS-CoV-nsp1-CD and pcDNA-MERS-CoV nsp1-CD, expressing a Cterminal myc-tagged MERS-CoV nsp 1 carrying the mutations R146A and K147A, were generated from pCAGGS-MERS-CoV-nsp1 and pcDNAMERS-nsp1, respectively, by using a recombinant PCR-based method. Sequence analyses of the plasmids confirmed the expected nspl sequences.
Generation of 293/DPP4 cells. A plasmid, pCAGGS-CD26-BlasticidinR, expressing the blasticidin resistance gene and the MERS-CoV receptor, human dipeptidyl peptidase-4 (DPP4; also known as CD26) was generated by replacing the coding region of Rift Valley fever virus (RVFV) Gn/Gc gene in pCAGGS-bla-G (26) with the human DPP4 gene from pcDL-SR $\alpha 296$ (27). 293 cells were transfected with pCAGGS-CD26BlasticidinR and grown in selection medium containing blasticidin (12 $\mu \mathrm{g} / \mathrm{ml}$ ) for 3 weeks. 293/DPP4 cells, stably expressing human DPP4, were selected based on the resistance to blasticidin. The expression of human DPP4 in 293/DPP4 cells was confirmed by Western blotting with antihuman DPP4 antibody (R\&D Systems).

Plasmid transfection, reporter assays, and Northern blot analysis. 293 cells, grown in 24-well plates, were cotransfected in triplicate with various combinations of plasmids $(1 \mu \mathrm{g}$ total) using the TransIT-293 reagent (Mirus). At $24 \mathrm{~h}$ posttransfection, cell lysates were prepared and subjected to Renilla luciferase (rLuc) reporter activity assays (Promega). For protein expression analysis by Western blotting, cell extracts were prepared in sodium dodecyl sulfate-polyacrylamide gel electrophoresis (SDS-PAGE) sample buffer. For RNA analysis, total RNAs were extracted, treated with DNase I, and subjected to Northern blot analysis with digoxigenin-labeled antisense rLuc RNA probe.

In vitro RNA transcription, RNA transfection, and RNA electroporation. Capped and polyadenylated RNA transcripts, encoding chloramphenicol acetyltransferase (CAT), SARS-CoV nsp1, MERS-CoV nsp1, or MERS-CoV nsp1-CD proteins, were synthesized from linearized plasmids or PCR products, encoding the respective genes, by using the mMESSAGE mMACHINE T7 Ultra kit (Ambion). The GLA and ALA reporter mRNAs were synthesized as described previously (20). To generate the MERS-CoV subgenomic mRNA 8-like RNA transcript, a PCR product carrying a T7 promoter upstream of a MERS-CoV mRNA 8-like sequence, encoding the viral nucleocapsid $(\mathrm{N})$ gene with a C-terminal V5 epitope tag flanked by the $5^{\prime}$ and $3^{\prime}$ untranslated regions (UTR) of MERSCoV mRNA 8 and a poly(A) tail, was used as the template. The PCR product was generated from cDNAs that were obtained from intracellular RNAs extracted from MERS-CoV-infected cells. The MERS-CoV subgenomic mRNA 8-like RNA transcript was synthesized from the PCR product by using the mMESSAGE mMACHINE T7 in vitro transcription kit. Subconfluent 293 cells, grown in 24 -well plates, were transfected with in vitro-synthesized RNA transcripts using the TransIT mRNA reagent (Mirus, Madison, WI). 293 cells were electroporated with the RNA transcripts using the Bio-Rad GenePulser Xcell electroporation system, according to the manufacturer's instructions.

Metabolic radiolabeling of intracellular proteins. Subconfluent 293 cells were transfected with in vitro-synthesized RNA transcripts and incubated either in a culture medium lacking actinomycin D (ActD) or containing $4 \mu \mathrm{g}$ of $\mathrm{ActD} / \mathrm{ml}$ from $1 \mathrm{~h}$ to $8 \mathrm{~h}$ posttransfection. Subsequently, the cells were starved for $30 \mathrm{~min}$ in methionine-deficient medium and metabolically labeled with $20 \mu \mathrm{Ci}$ of $\mathrm{Tran}^{35} \mathrm{~S}$-label (1,000 Ci/mmol; MP Biomedicals) $/ \mathrm{ml}$ for $1 \mathrm{~h}$. The cell extracts were prepared by lysing the cells in SDS-PAGE sample buffer, and equivalent amounts of the extracts were analyzed by SDS-PAGE. The radiolabeling of electroporated cells was performed at $24 \mathrm{~h}$ postelectroporation with $50 \mu \mathrm{Ci}$ of $\operatorname{Tran}{ }^{35} \mathrm{~S}$-label/ml for 1 h. MERS-CoV-infected 293/DPP4 cells were radiolabeled with $75 \mu \mathrm{Ci}$ of $\operatorname{Tran}^{35} \mathrm{~S}$-label/ml for $1 \mathrm{~h}$ at 18,24 , or $30 \mathrm{~h}$ postinfection (p.i.). The gels were visualized by autoradiography, and the band intensities in the selected regions of the gel were determined by densitometric scanning of the autoradiographs.

Western blot analysis. Western blot analysis was performed as described previously (14). Anti-MERS-CoV-nsp1 peptide antibody, generated by immunizing rabbits with the synthetic peptide (RKYGRGGYHY TPFHYERD), anti-myc mouse monoclonal antibody (MAb; Millipore) and anti-V5 rabbit MAb (Abcam) were used as primary antibodies. Goat anti-mouse IgG-horseradish peroxidase (HRP) and goat anti-rabbit IgGHRP (Santa Cruz Biotech) were used as secondary antibodies. 
Cosedimentation analysis. Cell lysates were prepared in a lysis buffer containing $50 \mathrm{mM}$ Tris- $\mathrm{HCl}$ ( $\mathrm{pH} 7.5$ ), $5 \mathrm{mM} \mathrm{MgCl}_{2}, 100 \mathrm{mM} \mathrm{KCl}, 1 \%$ (vol/vol) Triton X-100, $2 \mathrm{mM}$ dithiothreitol (DTT), $100 \mu \mathrm{g}$ of cycloheximide $/ \mu \mathrm{l}$, and $0.5 \mathrm{mg}$ of heparin/ $\mu \mathrm{l}$. The lysates were applied onto a 10 to $40 \%$ continuous sucrose gradient prepared in the same buffer and centrifuged at 38,000 rpm in a Beckman SW41 rotor at $4^{\circ} \mathrm{C}$ for $3 \mathrm{~h}$. After fractionation, the proteins in each fraction were precipitated with trichloroacetic acid-acetone and detected by Western blotting. Total RNAs were also extracted from the fractions, and the rRNAs were visualized by staining with ethidium bromide.

Confocal microscopy analysis. Cells, grown on chamber slides, were transfected with in vitro-transcribed RNA transcripts using the TransITmRNA reagent. At $16 \mathrm{~h}$ after transfection, the cells were fixed with $4 \%$ paraformaldehyde in phosphate-buffered saline (PBS) for $20 \mathrm{~min}$, permeabilized in PBS containing 0.5\% Triton X-100 for 15 min, blocked with PBS containing $3 \%$ bovine serum albumin for $30 \mathrm{~min}$, and immunostained with anti-V5 antibody (Abcam). The cells were examined under a Zeiss LSM 510 UV META laser scanning confocal microscope.

Preparation of cytoplasmic and nuclear extracts. 293/DPP4 cells were infected with MERS-CoV at a multiplicity of infection (MOI) of 3. At $18 \mathrm{~h}$ p.i., the cell suspension was prepared and frozen at $-80^{\circ} \mathrm{C}$ in the presence of dimethyl sulfoxide to preserve the integrity of the cell membrane. The frozen cells were irradiated with $2 \times 10^{6}$ rads from a Gammacell ${ }^{60}$ Co source (model 109A; J. L. Shepherd and Associates, San Fernando, CA) to completely inactivate MERS-CoV infectivity. After quickly thawing the frozen cells, cell lysates were prepared by incubating the cells in buffer 1 (25 mM HEPES [pH 7.9], $5 \mathrm{mM} \mathrm{KCl}, 0.5 \mathrm{mM} \mathrm{MgCl}_{2}, 1 \mathrm{mM}$ DTT, and $0.5 \%$ NP-40 supplemented with a protease inhibitor cocktail) for $15 \mathrm{~min}$ at $4^{\circ} \mathrm{C}$. After centrifugation at 5,000 rpm for $5 \mathrm{~min}$, the supernatants were collected and designated the cytoplasmic fractions. The pellets were incubated in buffer 2 ( $25 \mathrm{mM}$ HEPES [pH 7.9], $5 \mathrm{mM} \mathrm{KCl}, 0.5$ $\mathrm{mM} \mathrm{MgCl}, 1 \mathrm{mM}$ DTT, and $0.25 \% \mathrm{NP}-40$ supplemented with a protease inhibitor cocktail) for $10 \mathrm{~min}$ at $4^{\circ} \mathrm{C}$. After centrifugation, the pellets were collected and designated the nuclear fractions (28). Essentially, the same method was used to prepare the cytoplasmic and nuclear fractions from cells expressing MERS-CoV nsp1, except that the ${ }^{60} \mathrm{Co}$ irradiation step was omitted.

Generation of RVFV-like particles. RVFV virus-like particles (VLPs), carrying an RNA encoding the rLuc gene (LNCR-rLuc RNA) flanked by the $3^{\prime}$ and $5^{\prime}$ noncoding regions of RVFV L RNA, were prepared as described previously (29). Briefly, BSR-T7/5 cells (30), stably expressing T7 RNA polymerase, were cotransfected with a plasmid expressing T7 polymerase-driven RVFV antisense LNCR-rLuc RNA, along with the plasmids expressing L protein, $\mathrm{Gn} / \mathrm{Gc}$ envelope proteins, and N protein. VLPs carrying LNCR-rLuc RNA, released into the supernatant, were collected at 3 days posttransfection. 293 cells were electroporated with RNA transcripts encoding CAT, SARS-CoV nspl, MERS-CoV nsp1, or MERS-CoV nsp 1-CD proteins, and at $18 \mathrm{~h}$ postelectroporation, the cells were inoculated with RVFV VLPs. As a negative control, the cells were inoculated with UV-irradiated VLP. Cell extracts, prepared at $6 \mathrm{~h}$ post-VLP inoculation, were used for reporter assay and mRNA analysis.

Quantitative RT-PCR. Total cellular RNAs were extracted from VLPinfected cells by using TRIzol LS reagent (Invitrogen) and treated with RNase-free DNase I (Promega). cDNAs were synthesized using SuperScript III reverse transcriptase (Invitrogen) and an rLuc gene-specific primer, 5' -TTATTGTTCATTTTTGAGAACTCGC-3', for the quantification of rLuc mRNA and random primers for human 18S rRNA. Reverse transcription-PCR (RT-PCR) was performed using a Bio-Rad CFX96 real-time PCR apparatus and SYBR green Master mix (Bio-Rad). PCR conditions were as follows: preincubation at $95^{\circ} \mathrm{C}$ for $30 \mathrm{~s}$ and amplification with 40 cycles of $95^{\circ} \mathrm{C}$ for $15 \mathrm{~s}$ and $60^{\circ} \mathrm{C}$ for $20 \mathrm{~s}$. The purity of the amplified PCR products was confirmed by the dissociation melting curves obtained after each reaction. The primers used for rLuc mRNA were 5'-GCTTATCTACGTGCAAGTGATGATT-3' (forward) and 5' -TAGGA AACTTCTTGGCACCTTCAAC-3' (reverse); the primers for $18 \mathrm{~S}$ rRNA were 5' -CCGGTACAGTGAAACTGCGAATG-3' (forward) and 5' -GTT ATCCAAGTAGGAGAGGAGCGAG-3' (reverse). The relative levels of rLuc mRNA normalized to $18 \mathrm{~S}$ rRNA levels are presented in the data. All of the assays were performed in triplicate, and the results are expressed as means \pm the standard deviations.

\section{RESULTS}

MERS-CoV replication inhibits host protein synthesis and promotes host mRNA decay. As a first step toward exploring the role of MERS-CoV nsp1 in the regulation of host gene expression, we examined the effect of MERS-CoV replication on host protein synthesis and host mRNA stability in virus-infected cells. Metabolic radiolabeling experiments showed that MERS-CoV replication in 293/DPP4 cells, stably expressing the MERS-CoV receptor, human DPP4 (31), induced a strong inhibition of host protein synthesis, concomitant with an efficient production of virus-specific proteins, including nsp1 (Fig. 1A). MERS-CoV replication also caused a substantial reduction in the levels of endogenous GAPDH (glyceraldehyde-3-phosphate dehydrogenase) and $\beta$-actin mRNAs, and this effect was observed both in the absence and in the presence of actinomycin D (ActD), an inhibitor of host RNA transcription (Fig. 1B). Because ActD treatment prevents the synthesis of new RNAs, these data demonstrated that MERS-CoV replication induced the decay of preexisting host mRNAs in infected cells. Based on our previous studies with SARS-CoV nsp1 (32), these data strongly alluded to the possibility that nsp1 of MERS-CoV exerted these inhibitory effects on host gene expression in virus-infected cells.

MERS-CoV nsp1 inhibits host protein synthesis and induces endonucleolytic cleavage and degradation of mRNAs. To test the possibility that MERS-CoV nsp1 shares a common biological function with SARS-CoV nsp1 to inhibit host gene expression, we transfected 293 cells with RNA transcripts encoding CAT, MERSCoV nsp 1 or SARS-CoV nsp1 proteins. The transfected cells were incubated either in the presence or absence of ActD from $1 \mathrm{~h}$ posttransfection and metabolically radiolabeled with $\operatorname{Tran}^{35} \mathrm{~S}$-label from 8.5 to $9.5 \mathrm{~h}$. Cell extracts were prepared and subjected to SDS-PAGE, followed by Western blotting. The cells expressing CAT and SARS-CoV nsp1 served as negative and positive controls, respectively. Like SARS-CoV nsp1, MERS-CoV nsp1 also inhibited host protein synthesis both in the presence or absence of ActD (Fig. 2A). Densitometric analysis of the marked areas of the gels clearly showed that the band intensities of the radiolabeled host proteins in cells expressing SARS-CoV nsp 1 and MERS-CoV nsp1 were lower than those in cells expressing CAT (Fig. 2A). However, the extent of inhibition induced by SARS-CoV nsp1 was stronger than that induced by MERS-CoV nsp1.

To determine whether MERS-CoV nsp1 induces the degradation of endogenous host mRNAs, cells were transfected with RNA transcripts encoding CAT, MERS-CoV nsp1, or SARS-CoV nsp1 proteins and incubated in the presence of ActD from $1 \mathrm{~h}$ posttransfection. Intracellular RNAs were extracted at 1 and $9 \mathrm{~h}$ posttransfection and subjected to Northern blot analysis. Both MERS$\mathrm{CoV}$ nsp 1 and SARS-CoV nsp 1 expression caused a reduction in the levels of GAPDH and $\beta$-actin mRNAs (Fig. 2B). Like SARSCoV nsp1, MERS-CoV nsp1 also had no effect on the rRNA levels. Because ActD treatment prevents host RNA transcription, these data demonstrated that MERS-CoV nspl induced the degradation of preexisting host mRNAs.

SARS-CoV nsp1 induces an endonucleolytic RNA cleavage at 
A

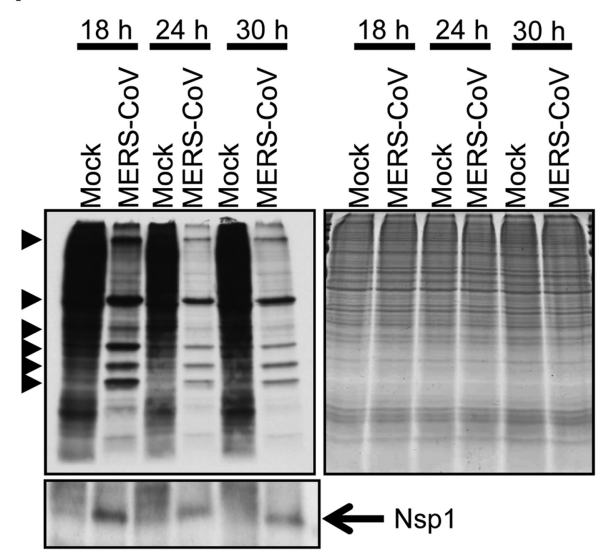

B

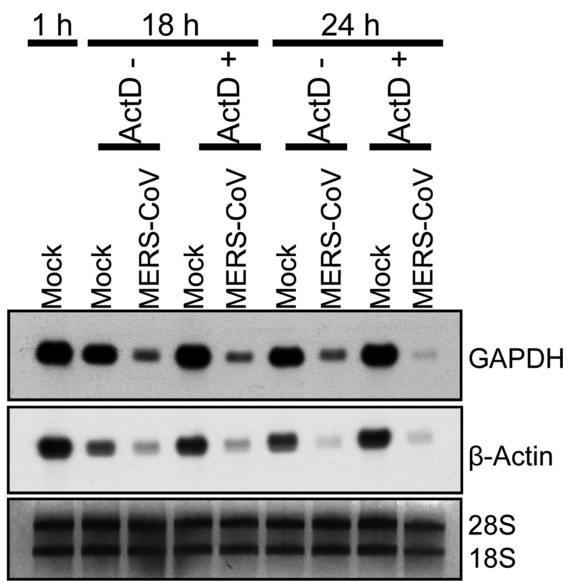

FIG 1 MERS-CoV replication inhibits host protein synthesis and induces host mRNA degradation. 293/DPP4 cells were mock infected or infected with MERS-CoV at an MOI of 3. (A) Cells were radiolabeled with $\operatorname{Tran}^{35} \mathrm{~S}$-label for $1 \mathrm{~h}$, and extracts were prepared at the indicated times postinfection (p.i.). Cell lysates were subjected to SDS-PAGE analysis, followed by autoradiography (top left panel), Western blot analysis with an anti-MERS-CoV-nsp1 peptide antibody (bottom left panel) and colloidal Coomassie blue staining (right panel). Arrowheads, MERS-CoV-specific proteins. (B) Cells were incubated in the absence or presence of ActD from $1 \mathrm{~h}$ p.i. Intracellular RNAs were extracted at 1, 18, 24, or $30 \mathrm{~h}$ p.i., and subjected to Northern blot analysis using a GAPDH mRNA-specific probe (top panel) and $\beta$-actin mRNA-specific probe (middle panel). The amounts of $28 \mathrm{~S}$ and $18 \mathrm{~S}$ rRNAs in each sample were detected by ethidium bromide staining (bottom panel).

the $5^{\prime}$ region of capped host mRNAs, as well as within the type I and II picornavirus internal ribosome entry sites (IRES), including those derived from encephalomyocarditis virus (EMCV) (14, 20). Subsequently, the endonucleolytically cleaved RNAs are rapidly degraded by the cellular exonuclease, Xrn1 (21). To determine whether MERS-CoV nsp1 exhibited a similar property to induce an endonucleolytic RNA cleavage in template mRNAs, cells were transfected with a plasmid encoding MERS-CoV nsp1, together with a plasmid encoding a bicistronic reporter mRNA carrying the EMCV IRES between the upstream Renilla luciferase (rLuc) gene and the downstream firefly luciferase (fLuc) gene (Fig. 2C). As controls, expression plasmids encoding CAT or SARS$\mathrm{CoV}$ nsp1 were used in place of MERS-CoV nsp1. Intracellular RNAs were extracted at $24 \mathrm{~h}$ posttransfection and subjected to Northern blot analysis. Cell extracts were also prepared at $24 \mathrm{~h}$ posttransfection and subjected to rLuc reporter assay. MERS-CoV nsp1 expression resulted in a marked reduction in the amount of the full-length Ren-EMCV-FF RNA, and the extent of reduction was similar to that induced by SARS-CoV nsp1 (Fig. 2C). Like SARS-CoV nsp1, MERS-CoV nsp1 expression also resulted in the generation of a cleaved RNA fragment derived from RenEMCV-FF with the same electrophoretic mobility as that observed with SARS-CoV nsp1 (Fig. 2C). As expected, this cleaved RNA fragment was not detected in cells expressing CAT. The amount of the cleaved RNA fragment was lower in MERS-CoV nsp1-expressing cells than in SARS-CoV nsp1-expressing cells. Based on our previous studies that have demonstrated that the cleaved RNA fragment detected in SARS-CoV nsp1-expressing cells is due to an endonucleolytic RNA cleavage at the ribosome loading site of EMCV IRES (20), our data strongly implied that MERS-CoV nsp1 also induced an endonucleolytic RNA cleavage at the ribosome loading site of EMCV IRES.

A SARS-CoV nsp1 mutant carrying alanine substitutions of two charged amino acid residues, R125 and K126, exposed on the surface of nsp1 (33), retained its ability to inhibit translation but lacked the endonucleolytic RNA cleavage function (19). Since the amino acid sequence alignment of MERS-CoV nsp1 with SARS$\mathrm{CoV}$ nsp 1 revealed two identical contiguous charged amino acids, R146 and K147, in MERS-CoV nsp1, we speculated that alanine substitutions of these two charged amino acids would similarly abolish the ability of MERS-CoV nsp1 to induce endonucleolytic RNA cleavage. Indeed, the expression of a mutated MERS-CoV nsp1, carrying R146A and K147A mutations (MERS-CoV nsp1$\mathrm{CD}$; the acronym $\mathrm{CD}$ stands for cleavage defective) neither induced the endonucleolytic RNA cleavage in Ren-EMCV-FF RNA nor caused a reduction in the abundance of the full-length RenEMCV-FF (Fig. 2C), demonstrating the lack of RNA cleavage activity in MERS-CoV nsp1-CD and the importance of these amino acid residues for the RNA cleavage function of MERS-CoV nsp1. Northern blot analysis of MERS-CoV nsp1 mRNA showed that the amount of expressed RNA encoding MERS-CoV nsp1 was lower than that encoding MERS-CoV nsp1-CD (Fig. 2C, second panel), suggesting that MERS-CoV nsp 1 targeted its own template mRNA for degradation and that MERS-CoV nsp1-CD lacked the ability to degrade mRNAs. Furthermore, MERS-CoV nsp1-CD expression did not cause a reduction in the amounts of GAPDH and $\beta$-actin mRNAs in the presence of ActD, suggesting that MERS-CoV nsp1-CD lacked the ability to induce the degradation of preexisting host mRNAs (Fig. 2B). These data point toward MERS-CoV nsp1-induced mRNA cleavage as the trigger that results in mRNA degradation.

Like SARS-CoV nsp1, MERS-CoV nsp 1 also strongly inhibited the rLuc reporter activity (Fig. 2C, fourth panel). It is important to note that MERS-CoV nsp1-CD also inhibited the rLuc reporter activity, albeit to a lesser extent than MERS-CoV nsp1 (Fig. 2C, fourth panel). Furthermore, metabolic radiolabeling experiments showed that MERS-CoV nsp1-CD expression inhibited host protein synthesis, but the extent of inhibition was lower than that induced by MERS-CoV nsp 1 (Fig. 2A). These data clearly demonstrated that the RNA cleavage function of MERS-CoV nsp1 contributed to, but was not required for, the ability of MERS-CoV nsp1 to inhibit host protein synthesis. 
A

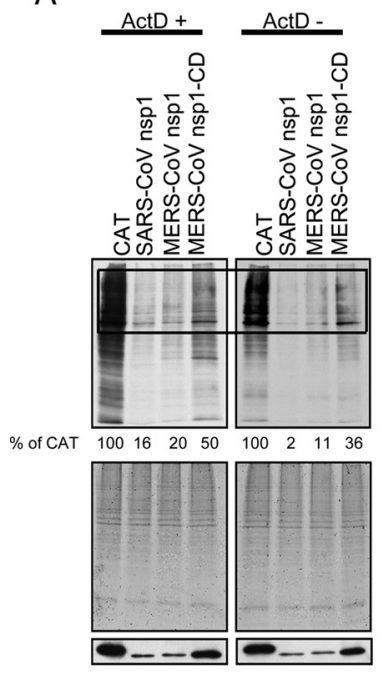

B

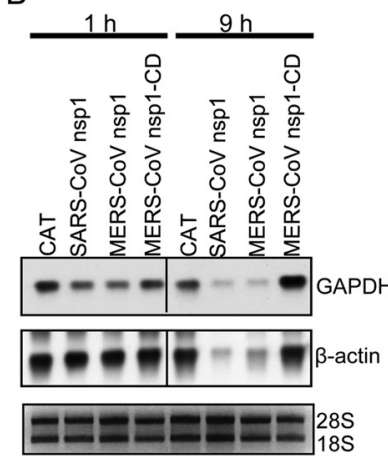

C

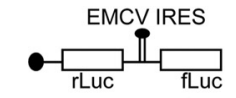

웅

할

웡
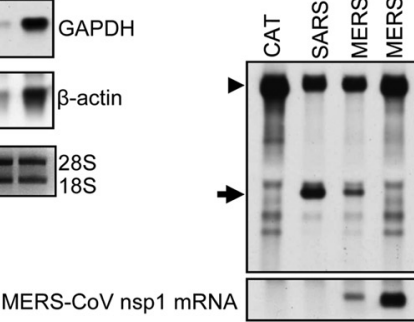

${ }_{185}==$

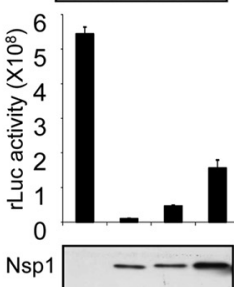

FIG 2 MERS-CoV nsp1 inhibits host protein synthesis and induces endonucleolytic cleavage and degradation of mRNAs. (A) 293 cells were transfected with RNA transcripts encoding CAT, SARS-CoV nsp1, MERS-CoV nsp1, or MERS-CoV nsp1-CD proteins, carrying a C-terminal myc epitope tag, and incubated in the absence or presence of ActD from $1 \mathrm{~h}$ posttransfection. Cells were radiolabeled with $\operatorname{Tran}^{35} \mathrm{~S}$-label from 8.5 to $9.5 \mathrm{~h}$ posttransfection, and lysates were resolved on SDS-12\% PAGE, followed by autoradiography (top panels), colloidal Coomassie blue staining (middle panels), and Western blot analysis using anti-myc antibody (bottom panels). Densitometric analysis of the autoradiographs was used to determine the levels of host protein synthesis, and the numbers below the lanes in the top panels represent percentage band intensity relative to CAT RNA-transfected cells (\% of CAT). The box represents the region of the gel used for densitometric analysis. Representative data from three independent experiments are shown. (B) 293 cells were transfected with RNA transcripts encoding CAT, SARS-CoV nsp1, MERS-CoV nsp1, or MERS-CoV nsp1-CD and incubated in the presence of ActD from $1 \mathrm{~h}$ posttransfection. At 1 and $9 \mathrm{~h}$ posttransfection, intracellular RNAs were extracted and subjected to Northern blot analysis with a GAPDH mRNA-specific probe (top panels) and $\beta$-actin mRNA-specific probe (middle panels). The bottom panel represents the amounts of $28 \mathrm{~S}$ and $18 \mathrm{~S}$ rRNAs in each sample. (C) 293 cells were cotransfected with a plasmid encoding Ren-EMCV-FF and the plasmid expressing CAT, SARS-CoV nsp1, MERS-CoV nsp1, or MERS-CoV nsp1-CD; the nsp1-expression plasmids encoded proteins carrying the C-terminal myc tag. At $24 \mathrm{~h}$ posttransfection, intracellular RNAs were extracted and subjected to Northern blot analysis using an RNA probe that binds to the rLuc gene (top panel) and MERS-CoV nsp1 gene (second panel), respectively. The $28 \mathrm{~S}$ and $18 \mathrm{~S}$ rRNAs were detected by ethidium bromide staining (third panel). Cell extracts, prepared at $24 \mathrm{~h}$ postelectroporation, were used for a reporter assay (fourth panel) and Western blot analysis, using an anti-myc antibody (bottom panel). Arrowhead, full-length Ren-EMCV-FF; arrow, cleaved RNA fragment. A schematic diagram of Ren-EMCV-FF RNA is shown at the top of the panel.

Taken together, these data suggest that MERS-CoV nsp1 possesses two distinct properties that exert an inhibitory effect on host gene expression: (i) the ability to promote the accelerated turnover of host mRNAs, by inducing an endonucleolytic RNA cleavage in template mRNAs, and (ii) a translation inhibitory function, which is separable from its RNA cleavage activity.

Subcellular localization of MERS-CoV nsp1 is different from SARS-CoV nsp1. Since both SARS-CoV nsp1 and MERS-CoV nsp1 exhibited similar inhibitory activities on host gene expression, we sought to determine whether the two proteins share a mode of action and identify any potential mechanistic differences between SARS-CoV nsp1 and MERS-CoV nsp1. To this end, we first examined the subcellular localization of MERS-CoV nsp1 in transfected cells, as well as in MERS-CoV-infected cells. Confocal microscopy (Fig. 3A) and subcellular fractionation analyses (Fig. 3B) showed the distribution of MERS-CoV nsp1 and MERS-CoV nsp1-CD in both the cytoplasm and the nuclei of transfected cells expressing nsp1. Importantly, subcellular fractionation analysis of MERS-CoV-infected cells, using an anti-MERS-CoV-nsp1 pep- tide antibody, showed a similar distribution pattern of MERS$\mathrm{CoV}$ nsp1 in both the cytoplasm and the nuclei of MERS-CoVinfected cells (Fig. 3C). The purity of the cytoplasmic and nuclear fractions was validated by Western blotting of the subcellular fractions using anti-GAPDH and anti-histone $\mathrm{H} 3$ antibodies, respectively (Fig. 3B and C). The distribution of MERS-CoV nsp1 in both the cytoplasm and the nucleus was in marked contrast to the localization of SARS-CoV nsp1 exclusively in the cytoplasm $(11,15)$.

MERS-CoV nsp1 and SARS-CoV nsp1 use different strategies to target translationally competent mRNAs for degradation. The subcellular distribution of MERS-CoV nsp 1 in both the cytoplasm and nucleus prompted us to investigate whether MERS-CoV nsp1 also targets RNAs transcribed by nuclear RNA polymerase I (Pol I) and Pol III for degradation. Cells were transfected with the plasmid encoding MERS-CoV nsp1, together with either both Pol II-driven reporter plasmid expressing green fluorescent protein (GFP) mRNA and Pol III-driven GFP plasmid or a Pol I-driven GFP reporter plasmid; the Pol I- and Pol III-driven 
A
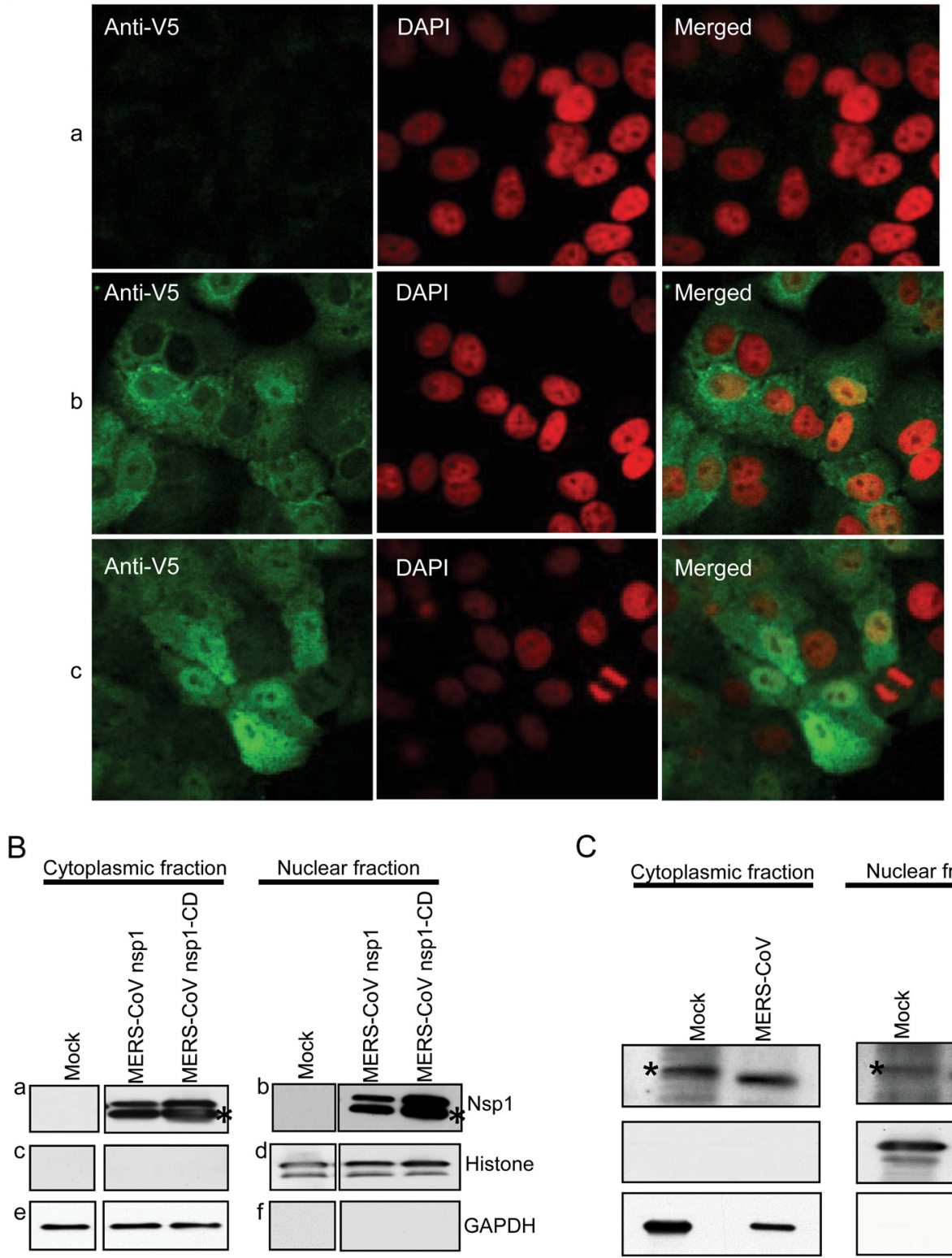

C

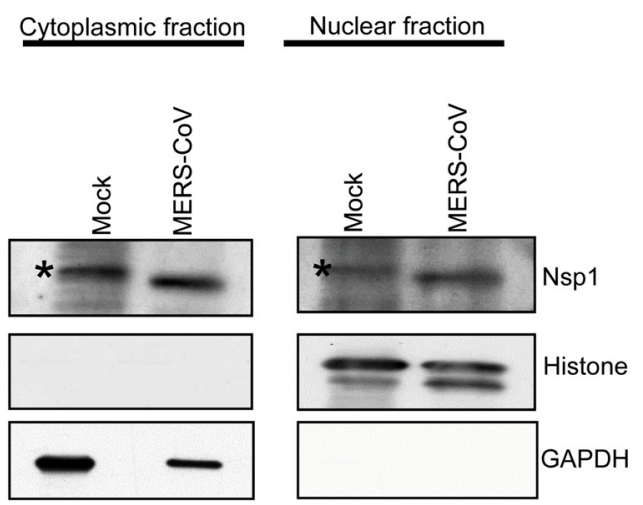

FIG 3 Subcellular distribution of MERS-CoV nsp1 in both the nucleus and the cytoplasm. (A) Vero E6 cells were mock transfected (a) or transfected with RNA transcripts encoding C-terminal V5-tagged MERS-CoV nsp1 (b) or MERS-CoV nsp1-CD (c). At $16 \mathrm{~h}$ after transfection, the cells were fixed, permeabilized, and subjected to immunofluorescence analysis with an anti-V5 antibody (Anti-V5). The nuclei were counterstained with 4',6-diamidino-2-phenylindole (DAPI), and the images were examined using a Zeiss LSM 510 UV META laser scanning confocal microscope. Merged images are shown in the rightmost panels. (B) 293 cells were mock transfected (Mock) or transfected with a plasmid encoding the C-terminal myc-tagged MERS-CoV nsp1 (MERS-CoV nsp1) or MERS-CoV nsp1-CD (MERS-CoV nsp1-CD). At $18 \mathrm{~h}$ posttransfection, cell lysates were fractionated into cytoplasmic and nuclear fractions. Subsequently, the fractions were subjected to Western blot analysis with anti-myc (top panels), anti-histone H3 (middle panels), and anti-GAPDH antibodies (bottom panels). The asterisks in the top panels represent possible proteolytic cleavage products of MERS-CoV nspl and MERS-CoV nspl-CD, probably generated during sample preparation. (C) 293/DPP4 cells were mock infected (Mock) or infected with MERS-CoV at an MOI of 3 (MERS-CoV). At $18 \mathrm{~h}$ p.i., the cell suspension was irradiated with ${ }^{60} \mathrm{Co}$ to inactivate MERS-CoV, and cell extracts were separated into cytoplasmic and nuclear fractions. Each fraction was subjected to Western blot analysis with anti-MERS-CoV-nspl peptide antibody (top panels), anti-histone $\mathrm{H} 3$ antibody (middle panels), or anti-GAPDH antibody (bottom panels). The asterisks (mock-infected cell extracts, top panels) represent a host protein with a slower migration than nsp1 in the gel that is recognized nonspecifically by the anti-MERS-CoV-nsp1 peptide antibody.

reporter plasmids encode a truncated GFP. As controls, plasmids encoding CAT or SARS-CoV nsp1 were used in place of the MERS-CoV nsp1 expression plasmid. Consistent with a previous report (21), SARS-CoV nsp1 expression induced the degradation of only the Pol II-driven transcripts, but not the Pol I- or Pol
III-driven transcripts (Fig. 4A). Similarly, MERS-CoV nsp1 expression also induced the degradation of only the Pol II-driven transcripts but did not affect the levels of the Pol I- or Pol IIIdriven transcripts (Fig. 4A). A minor band migrating below the Pol III-driven transcript was also observed in a published study 


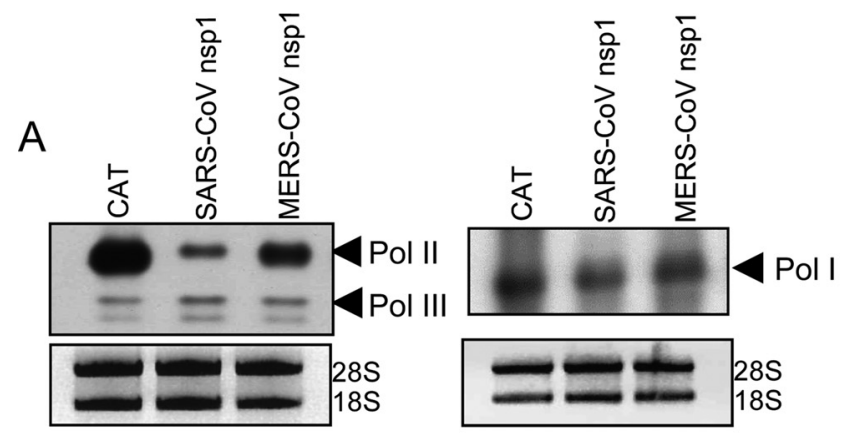

B
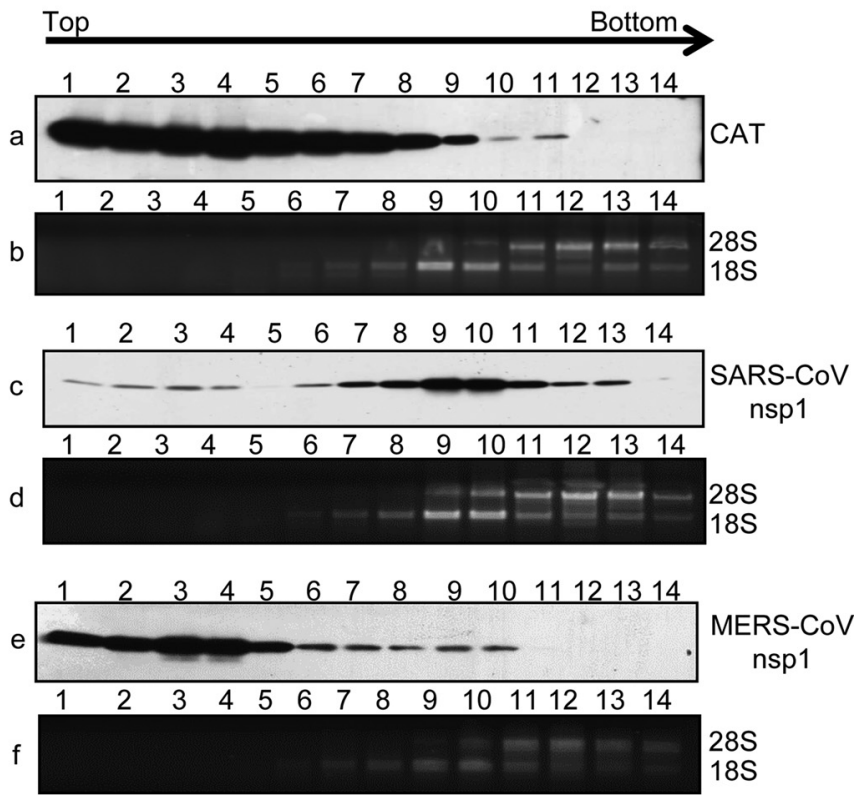

FIG 4 MERS-CoV nsp1 targets translationally competent Pol II-transcribed mRNAs for degradation but does not cosediment with 40S ribosomal subunits. (A) 293 cells were transfected with a plasmid encoding CAT, SARS-CoV nsp1, or MERS-CoV nsp1, together with Pol II- and Pol III-driven reporter plasmids encoding GFP RNA or a Pol I-driven GFP reporter plasmid. At $24 \mathrm{~h}$ posttransfection, the RNAs were extracted, treated with DNase I, and visualized by Northern blotting with a GFP-specific probe. The $28 \mathrm{~S}$ and $18 \mathrm{~S}$ rRNAs were detected by ethidium bromide staining (bottom panels). (B) 293 cells were transfected with RNA transcripts encoding C-terminal myc-tagged CAT (panels a and b), SARS-CoV nspl (panels c and d), or MERS-CoV nsp1 (panels $\mathrm{e}$ and $\mathrm{f}$ ). Cell extracts, prepared at $8 \mathrm{~h}$ posttransfection, were subjected to sucrose gradient centrifugation analysis. The gradient fractions were analyzed by Western blotting with anti-myc antibody to detect the expressed proteins (panels a, c, and e) and ethidium bromide staining to detect rRNAs (panels b, $\mathrm{d}$, and $\mathrm{f}$ ).

using the same plasmid (21). The source of this band is unknown. These data suggested that like SARS-CoV nsp1, MERS-CoV nsp1 also targets RNAs that are translationally competent for degradation.

SARS-CoV nspl targets translating mRNAs for mRNA cleavage and translation inhibition by binding to the $40 \mathrm{~S}$ ribosomal subunit (14). To evaluate whether MERS-CoV nspl adopted a similar strategy to gain access to translating cellular mRNAs, we examined the association of MERS-CoV nsp1 with 40S subunits by sucrose gradient sedimentation analysis of extracts from 293 cells expressing MERS-CoV nsp1. Lysates from cells expressing
CAT or SARS-CoV nsp1 served as negative and positive controls, respectively. In agreement with our previous studies (14), SARSCoV nsp1 tightly associated with the $40 \mathrm{~S}$ subunit as demonstrated by the cosedimentation of SARS-CoV nsp1 with the $40 \mathrm{~S}$ peak (determined by detecting $18 \mathrm{~S}$ rRNA, a component of the $40 \mathrm{~S}$ ribosomal subunit) (Fig. 4B). In marked contrast, the sedimentation profile of MERS-CoV nsp1 was very different from SARSCoV nsp1 and mirrored the profile observed for CAT. Most of the MERS-CoV nsp1 was detected near the top of the gradient and did not cosediment with the $40 \mathrm{~S}$ subunit, suggesting that unlike SARS-CoV nsp1, MERS-CoV nsp1 does not associate tightly with the $40 \mathrm{~S}$ subunit and uses a different strategy to gain access to translationally competent mRNAs (Fig. 4B).

The translation inhibitory activity of MERS-CoV nsp1 specifically targets nuclear-transcribed mRNAs but spares mRNAs that enter across the cytoplasmic membrane. The lack of binding of MERS-CoV nsp 1 to the $40 \mathrm{~S}$ subunit combined with its subcellular distribution in both the nucleus and the cytoplasm led us to examine whether the translation inhibitory activity of MERS-CoV nsp1 selectively targets mRNAs of nuclear origin and spares mRNAs that enter across the cytoplasmic membrane.

To test the effect of MERS-CoV nspl on the translation of nuclear-transcribed mRNAs, 293 cells were transfected with the plasmid encoding CAT, MERS-CoV nsp1, MERS-CoV nsp1-CD, or SARS-CoV nsp1, together with a reporter plasmid encoding the rLuc gene, and assayed for luciferase reporter activity at $24 \mathrm{~h}$ posttransfection. Intracellular RNAs were also extracted at $24 \mathrm{~h}$ posttransfection and subjected to Northern blot analysis. As expected, SARS-CoV nspl strongly inhibited the reporter gene activity and induced the degradation of rLuc mRNA (Fig. 5A). MERS-CoV nsp 1 also strongly inhibited the reporter gene activity and induced the degradation of rLuc mRNA (Fig. 5A), which is consistent with our data in Fig. 2 that showed the inhibition of host protein synthesis and induction of reporter mRNA cleavage and degradation by MERS-CoV nsp1. MERS-CoV nsp1-CD did not promote the degradation of rLuc mRNA but inhibited the reporter gene activity, albeit to a slightly lesser extent than MERS-CoV nsp1, further confirming that MERS-CoV nsp1-CD can inhibit translation without inducing mRNA cleavage (Fig. 5A). Collectively, these data unambiguously demonstrated that MERS-CoV nsp1 inhibited the translation and induced the degradation of reporter mRNAs as well as cellular mRNAs that are transcribed in the nucleus and transported to the cytoplasm (Fig. 2, 4A, and 5A).

To examine the effect of MERS-CoV nsp 1 on the translation of exogenous mRNAs introduced directly into the cytoplasm, 293 cells were electroporated with a reporter mRNA, GLA, carrying the $5^{\prime}$ UTR of rabbit $\beta$-globin mRNA and the rLuc gene (20), together with RNA transcripts encoding CAT, SARS-CoV nsp1, MERS-CoV nsp1, or MERS-CoV nsp1-CD; all of the transcripts were capped and polyadenylated. Cell extracts, prepared at $24 \mathrm{~h}$ postelectroporation, were subjected to luciferase reporter assays and Western blot analysis. SARS-CoV nspl expression inhibited the reporter gene activity (Fig. 5B), a result that is consistent with the ability of SARS-CoV nsp1 to load onto translating mRNAs, through its association with the $40 \mathrm{~S}$ subunit, leading to translation inhibition and degradation of the electroporated GLA RNA. Strikingly, both MERS-CoV nsp1 and MERS-CoV nsp1-CD did not inhibit the luciferase reporter activity (Fig. 5B). Instead, the reporter activity in cells expressing MERS-CoV nspl was higher than in cells expressing CAT and MERS-CoV nsp1-CD (Fig. 5B). 

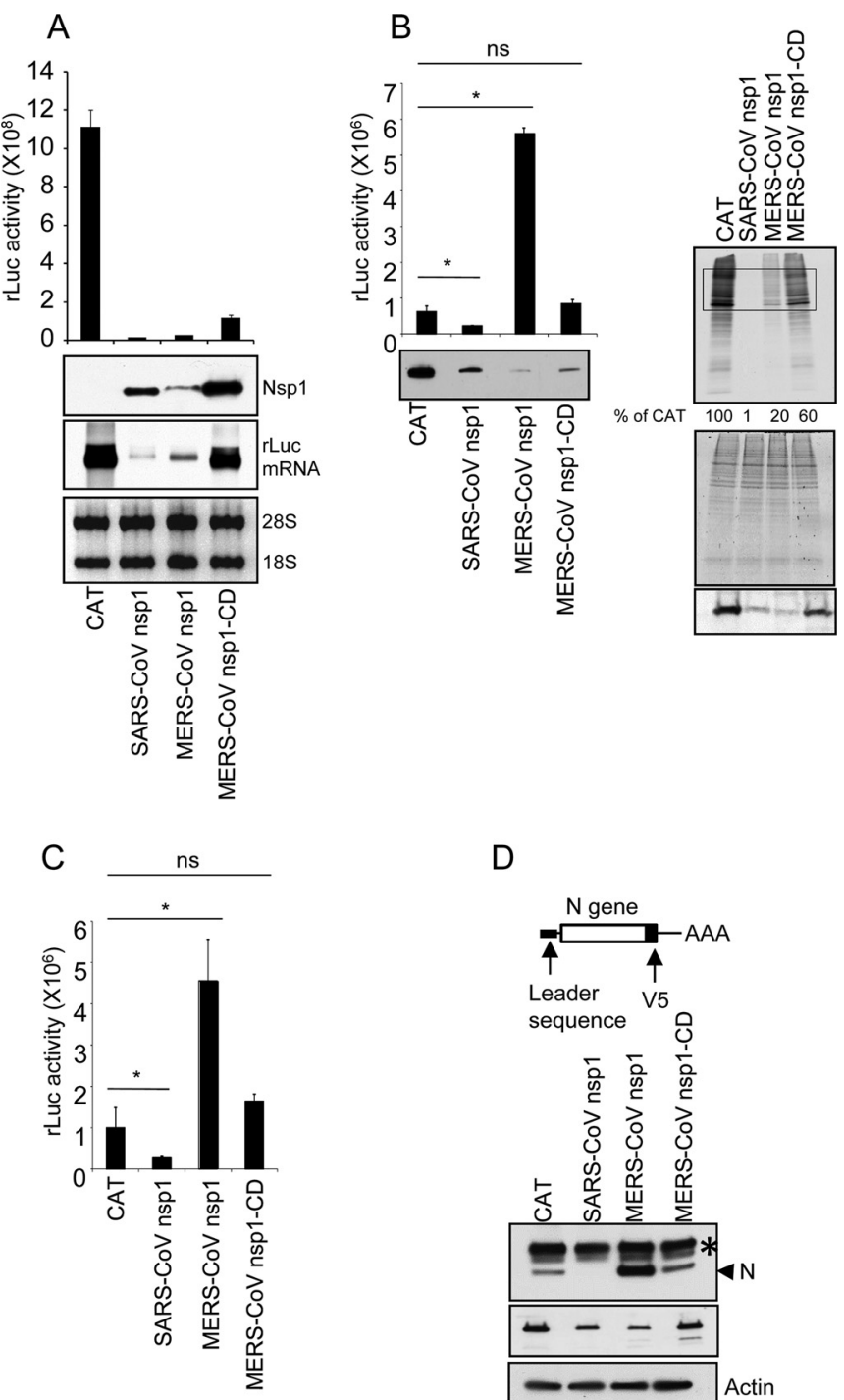

$\mathrm{D}$

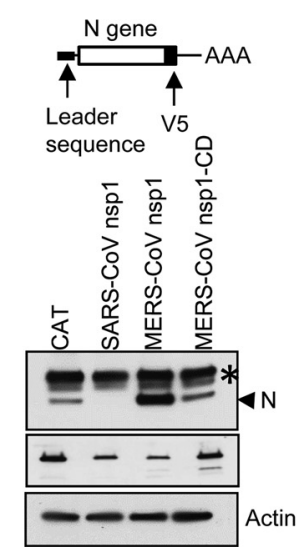

FIG 5 MERS-CoV nsp1 does not inhibit the translation of mRNAs introduced directly into the cytoplasm. (A) 293 cells were transfected with the reporter plasmid pRL-SV40 (15) encoding the rLuc gene, together with the expression plasmids encoding CAT, SARS-CoV nsp1, MERS-CoV nsp1, or MERS-CoV nsp1-CD; the nsp1 proteins had a C-terminal myc tag. Cell extracts, prepared at $24 \mathrm{~h}$ posttransfection, were used for a reporter assay (top panel) and Western blot analysis, using anti-myc antibody (second panel). Intracellular RNAs were also extracted at $24 \mathrm{~h}$ posttransfection and subjected to Northern blot analysis using the RNA probe that binds to the rLuc gene (third panel). The $28 \mathrm{~S}$ and $18 \mathrm{~S}$ rRNAs were detected by ethidium bromide staining (bottom panel). Representative data from three independent experiments are shown. (B) 293 cells were coelectroporated with GLA reporter mRNA and RNA transcripts encoding C-terminal myc-tagged CAT, SARSCoV nsp1, MERS-CoV nsp1, or MERS-CoV nsp1-CD. Cell extracts, prepared at $24 \mathrm{~h}$ postelectroporation, were used for the reporter assay (left top panel) and Western blot analysis, using anti-myc antibody (left bottom panel). Representative data from three independent experiments is shown. Asterisks indicate statistically significant differences between samples $(P<0.01)$; ns, not significant $(P>0.01)$. Cells that were electroporated with the RNA transcripts were also radiolabeled with $\operatorname{Tran}^{35} \mathrm{~S}$-label for $1 \mathrm{~h}$ at $24 \mathrm{~h}$ postelectroporation, and lysates were resolved on SDS-PAGE, followed by autoradiography (right top panel), colloidal Coomassie blue staining (right middle panel), and Western blot analysis with anti-myc antibody (right bottom panel). Densitometric analysis of the autoradiographs was used to determine the levels of host protein synthesis, and the numbers below the lanes in the right top panel represent the percent band intensity relative to CAT RNA-transfected cells ( $\%$ of CAT). The
Metabolic radiolabeling experiments and densitometric analysis of the marked areas of the gel clearly showed that SARS-CoV nsp1, MERS-CoV nsp1, and MERS-CoV nsp1-CD inhibited host protein synthesis, validating the inhibitory activity of the expressed nsp1 proteins toward the translation of nucleus-derived cellular mRNAs (Fig. 5B). We observed slight differences in the levels of accumulation of MERS-CoV nsp1 and MERS-CoV nsp1-CD, which could possibly be due to differences in the inherent stability of the two proteins (Fig. 5B). We obtained similar results using a different reporter mRNA, ALA, carrying the $5^{\prime}$ UTR of $\beta$-actin mRNA and the rLuc gene (20), wherein both MERS-CoV nsp1 and MERS-CoV nsp1-CD did not inhibit the reporter gene activity and an increased reporter activity was observed in cells expressing MERS-CoV nsp1 (Fig. 5C).

Since MERS-CoV mRNAs are cytoplasmic in origin, we tested the effect of MERS-CoV nsp1 on the translation of a MERS-CoVlike mRNA that was introduced directly into the cytoplasm. 293 cells were electroporated with a MERS-CoV subgenomic mRNA 8-like RNA transcript, carrying the $\mathrm{N}$ gene open reading frame (ORF) with a C-terminal V5 epitope tag flanked by the authentic 5' and 3' UTRs of mRNA 8 (Fig. 5D), together with RNA transcripts encoding CAT, SARS-CoV nsp1, MERS-CoV nsp1, or MERS-CoV nsp1-CD. Cell extracts, prepared at $24 \mathrm{~h}$ postelectroporation, were subjected to Western blot analysis to examine the expression level of $\mathrm{N}$ protein. SARS-CoV nsp1 strongly inhibited the expression of $\mathrm{N}$ protein (Fig. 5D). In contrast, both MERSCoV nsp1 and MERS-CoV nsp1-CD did not inhibit the expression of $\mathrm{N}$ protein and the level of $\mathrm{N}$ protein was higher in cells expressing MERS-CoV nsp1 than in cells expressing CAT and MERS-CoV nsp1-CD.

Taken together, these data demonstrated that MERS-CoV nsp1 did not inhibit the translation of exogenous reporter mRNAs and MERS-CoV-like mRNA that were introduced directly into the cytoplasm. Furthermore, MERS-CoV nsp1, but not MERSCoV nsp1-CD, had a positive effect on the translation of these mRNAs, suggesting the indirect role of its RNA cleavage function in this activity.

MERS-CoV nsp1 does not inhibit the translation of viruslike mRNAs synthesized in the cytoplasm. We further extended our findings to evaluate whether MERS-CoV nsp1 also spared mRNAs that are cytoplasmic in origin from its translation inhibitory activity by examining the effect of MERS-CoV nsp 1 on the translation of virus-like mRNAs synthesized in the cytoplasm. We used VLPs derived from RVFV (family Bunyaviridae, genus Phlebovirus), a cytoplasmic RNA virus, as the vehicle for the synthesis

box represents the region of the gel used for densitometric analysis. Representative data from three independent experiments is shown. (C) RNA coelectroporation and reporter assay were performed as described in panel B, except that ALA reporter mRNA was used in place of the GLA mRNA. Representative data from three independent experiments are shown. Asterisks indicate statistically significant differences between samples $(P<0.01)$; ns, not significant $(P>0.01)$. (D) RNA coelectroporation was performed as described in panel B, except that a MERS-CoV subgenomic mRNA 8-like RNA transcript, expressing a C-terminal V5-tagged MERS-CoV N protein, was used in place of the GLA mRNA. Cell extracts, prepared at $24 \mathrm{~h}$ postelectroporation, were subjected to Western blot analysis with anti-V5 antibody (top panel), anti-myc antibody (middle), and anti-actin antibody (bottom). The asterisk in the top panel represents a host protein that is recognized nonspecifically by the anti-V5 antibody. A schematic diagram of the MERS-CoV subgenomic mRNA 8-like RNA transcript is shown on top. 
A

Generation of RVFV VLP carrying a virus-like RNA coding for a reporter gene

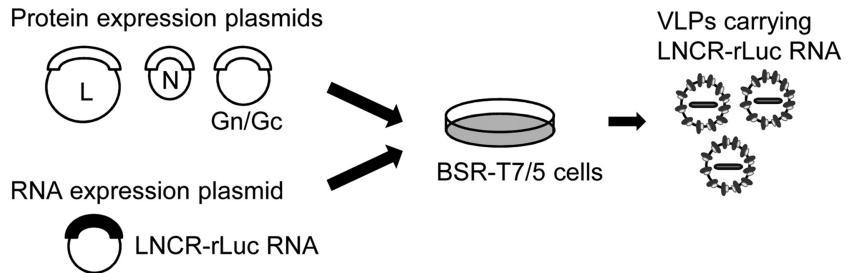

Testing the effect of nsp1 on the translation of mRNA synthesized in the cytoplasm

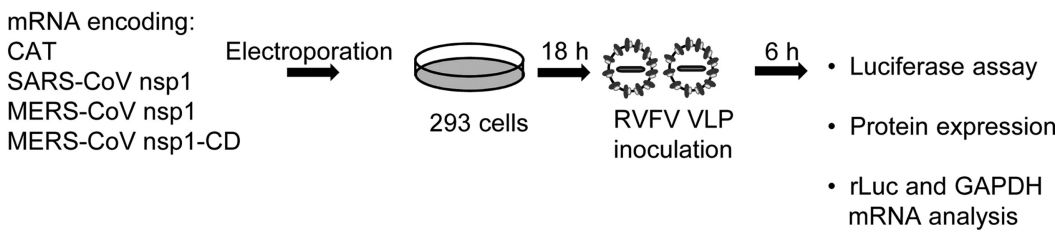

B

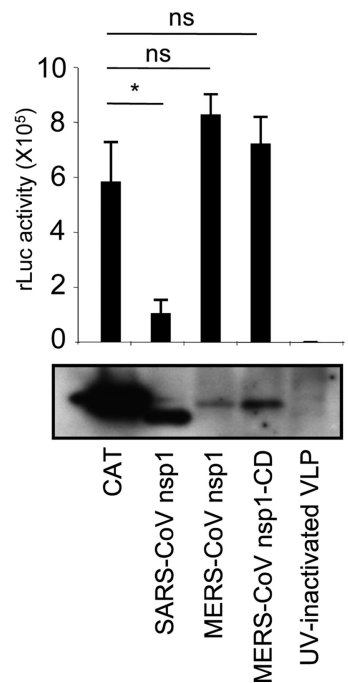

C

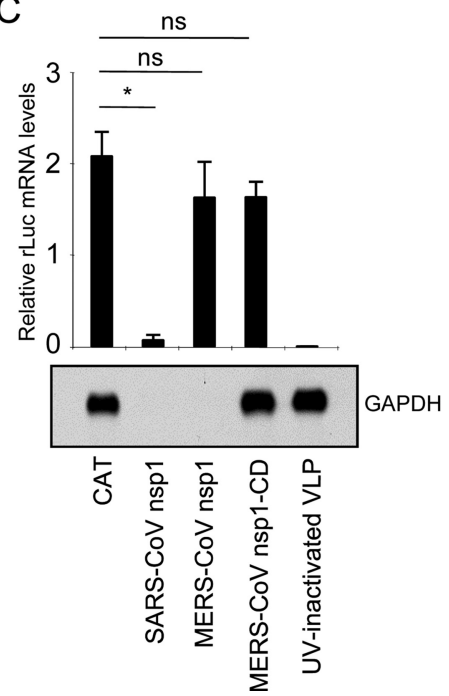

FIG 6 MERS-CoV nsp1 does not inhibit the translation of virus-like mRNAs synthesized in the cytoplasm (A) Schematic diagram of the experimental approach. BSR-T7/5 cells, stably expressing T7 RNA polymerase, were cotransfected with a plasmid expressing T7 polymerase-driven RVFV antisense LNCR-rLuc RNA, along with the plasmids expressing L protein, Gn/Gc envelope proteins and N protein. VLPs carrying LNCR-rLuc RNA, released into the supernatant, were collected at 3 days posttransfection. 293 cells were electroporated with RNA transcripts encoding CAT, SARS-CoV nsp1, MERS-CoV nsp1, or MERS-CoV nsp1-CD proteins and, at $18 \mathrm{~h}$ postelectroporation, the cells were inoculated with RVFV VLPs. As a negative control, cells were inoculated with UV-irradiated VLP. Cell extracts, prepared at $6 \mathrm{~h}$ post-VLP inoculation, were used for reporter assay, protein expression, and mRNA analyses. (B) The top panel shows the luciferase reporter activities at $6 \mathrm{~h}$ post-VLP inoculation. The bottom panel represents the Western blot analysis with anti-myc antibody. (C) Intracellular RNAs were extracted at $6 \mathrm{~h}$ post-VLP inoculation and subjected to qRT-PCR to determine the levels of LNCR-rLuc mRNA and $18 \mathrm{~S}$ rRNA. The plot shows the relative levels of LNCR-rLuc mRNA normalized to $18 \mathrm{~S}$ rRNA levels. The bottom panel shows a Northern blot analysis of GAPDH mRNA. Error bars in the plot represent standard deviations of three independent experiments. Asterisks indicate statistically significant differences between samples $(P<0.01)$; ns, not significant $(P>0.01)$.

of virus-like mRNAs in the cytoplasm. RVFV carries a tripartite, single-stranded, negative-sense RNA genome composed of $\mathrm{L}, \mathrm{M}$, and S RNA segments (34). We generated RVFV VLPs, carrying a single RNA segment, LNCR-rLuc RNA, encoding the rLuc reporter gene flanked by the $3^{\prime}$ and $5^{\prime}$ noncoding regions of RVFV L RNA, from cells expressing LNCR-rLuc RNA, L protein, N protein and the envelope $\mathrm{Gn} / \mathrm{Gc}$ proteins, as described previously (Fig. 6A) (29, 35). Inoculation of the RVFV VLPs into susceptible cells results in the synthesis of LNCR-rLuc mRNAs, carrying the rLuc ORF, in the cytoplasm, due to primary transcription from the incoming virion-associated LNCR-rLuc RNA mediated by the virion-associated $\mathrm{L}$ and $\mathrm{N}$ proteins. However, subsequent RNA replication and secondary transcription from LNCR-rLuc RNA does not occur in VLP-inoculated cells in the absence of de novo synthesis of $L$ and $N$ proteins (35). To examine the effect of MERSCoV nsp1 on the translation and stability of cytoplasmically synthesized LNCR-rLuc mRNA, 293 cells were electroporated with RNA transcripts encoding CAT, SARS-CoV nsp1, MERS-CoV nsp1 or MERS-CoV nsp1-CD. At $18 \mathrm{~h}$ postelectroporation, the cells were inoculated with RVFV VLP, carrying LNCR-rLuc RNA. As a negative control, cells were inoculated with UV-irradiated VLPs. Cell extracts, prepared at $6 \mathrm{~h}$ post-VLP inoculation, were 
used for luciferase reporter assay, Western blot analysis, to confirm the expression of proteins from the electroporated mRNAs, and the quantification of LNCR-rLuc mRNA levels by qRT-PCR. As expected, very low background levels of luciferase reporter activity and LNCR-rLuc mRNA were detected in cells inoculated with the UV-irradiated VLP (Fig. 6B and C). The luciferase reporter activity (Fig. 6B) and LNCR-rLuc mRNA levels (Fig. 6C) were substantially lower in cells expressing SARS-CoV nsp 1 than in cells expressing CAT, demonstrating that SARS-CoV nsp1 was able to target the cytoplasmically synthesized LNCR-rLuc mRNA for translation inhibition and RNA degradation. In contrast, there was no statistically significant difference in the reporter activity and LNCR-rLuc mRNA levels between cells expressing CAT, MERS-CoV nsp1 and MERS-CoV nsp1-CD, demonstrating that MERS-CoV nsp1 did not affect the translation and stability of cytoplasmically synthesized LNCR-rLuc mRNA (Fig. 6B and C). Both SARS-CoV nsp1 and MERS-CoV nsp1 induced the degradation of endogenous nucleus-derived GAPDH mRNA, confirming their biologically activity (Fig. 6C). These observations further bolster the idea that MERS-CoV nsp1 specifically targets the nuclear-transcribed host mRNAs for inhibition, and mRNAs, including MERS-CoV mRNAs, which are cytoplasmic in origin, are spared from its inhibitory effects.

\section{DISCUSSION}

In the present study, we first examined the effect of MERS-CoV infection on host gene expression that showed similarities between MERS-CoV and another highly pathogenic human CoV, SARS-CoV, in exerting an inhibitory effect on host gene expression at the level of translation (15) (Fig. 1). This observation and our prior knowledge of the properties of SARS-CoV nsp1 led us to investigate whether MERS-CoV nspl shared a common function to inhibit host gene expression by targeting mRNA translation and stability (32). A functional comparison between MERS-CoV nsp1 and SARS-CoV nsp1 showed some common features but also revealed intriguing differences in their mechanism of action. Like SARS-CoV nsp1, MERS-CoV nsp1 also exhibited two distinct properties that leads to the inhibition of host gene expression: (i) the ability to promote the degradation of host mRNAs, by inducing an endonucleolytic RNA cleavage in template mRNAs, and (ii) inhibition of host mRNA translation, a function that is separable from its RNA cleavage activity (Fig. 2 and 5A). Also, both MERSCoV nsp1 and SARS-CoV nsp1 only targeted RNAs that are translationally competent for degradation (Fig. 4A).

However, unlike SARS-CoV nsp1, which is localized exclusively in the cytoplasm $(11,15)$, MERS-CoV nsp1 was distributed in both the nucleus and cytoplasm (Fig. 3). Nsp1 ( $\sim 9 \mathrm{kDa})$ of transmissible gastroenteritis virus (TGEV), an alpha $\mathrm{CoV}$, is also distributed in both the nucleus and cytoplasm of transfected cells expressing nsp1 (36). Although TGEV nsp1 shares a common biological function with SARS-CoV nsp 1 and MERS-CoV nsp1 to inhibit host gene expression, it lacks the activity to induce host mRNA degradation (16). Analysis of the primary amino acid sequence of MERS-CoV nsp1 did not reveal any nuclear localization signal. Although, MERS-CoV nspl could diffuse into the nucleus because of its low molecular mass $(\sim 20 \mathrm{kDa})$, which is below the size exclusion limit of the nuclear pore complex, it is also possible that its nuclear accumulation could also be attributed to binding to a component of the nucleus after entry by diffusion. There are examples of small viral proteins that localize to the nucleus despite lacking any defined nuclear localization signal $(37,38)$. Importantly, the different subcellular distribution profiles of MERSCoV nsp1 and SARS-CoV nsp1 hinted at a possible fundamental difference between their mechanisms of action. Indeed, while SARS-CoV nsp1 associates tightly with the 40 S ribosomal subunit, MERS-CoV nsp1 did not cosediment with the 40S subunit, indicating the lack of stable binding to the $40 \mathrm{~S}$ subunit (Fig. 4A). These data suggested that unlike SARS-CoV nsp1, which gains access to translating mRNAs by binding to the 40S subunit (14), MERSCoV nspl uses a different strategy to target translationally competent mRNAs.

Further evidence in support of the idea that MERS-CoV nsp1 employs a different strategy to gain access to translationally competent mRNAs was provided by the striking observation that MERS-CoV nsp1 selectively inhibited the translation of nucleartranscribed mRNAs but did not inhibit the translation of mRNAs that enter across the cytoplasmic membrane or are synthesized in the cytoplasm (Fig. 5 and 6). In contrast, SARS-CoV nsp1 inhibited the translation of both nucleus-derived mRNAs and mRNAs of cytoplasmic origin (Fig. 5 and 6). These data are consistent with the observations that SARS-CoV nsp 1 targets translating mRNAs through its association with the $40 \mathrm{~S}$ subunit, a core component of the cellular translation apparatus (14), and MERS-CoV nsp1 does not bind to the $40 \mathrm{~S}$ subunit (Fig. 4A). The inability of MERS-CoV nsp1 to inhibit the translation of mRNAs of cytoplasmic origin suggests that MERS-CoV nsp1 does not utilize the components of the core translational machinery to gain access to translating mRNAs. In addition, it also indicates that MERS-CoV nsp1 does not affect the functions of these components involved in the translation of such mRNAs.

MERS-CoV nsp1 displayed an intriguing property to selectively target mRNAs, which are transcribed in the nucleus and transported to the cytoplasm, for translation inhibition and mRNA degradation (Fig. 2, 4A, and 5). Interestingly, MERS-CoV nsp1 inhibited host protein synthesis and induced the degradation of endogenous host mRNAs even in the presence of ActD, which prevents the synthesis of new mRNAs (Fig. 2A and B). These data suggested that the inhibitory activity of MERS-CoV nsp1 on nucleus-derived mRNAs is not exclusively restricted to newly synthesized mRNAs and can also target preexisting nuclear-transcribed mRNAs in the cytoplasm. The activity of MERS-CoV nsp1 was directed toward different nuclear-transcribed mRNAs, including endogenous host mRNAs and plasmid-driven reporter mRNAs. Eukaryotic mRNAs that are transcribed in the nucleus are transported to the cytoplasm in the form of an $\mathrm{mRNP}$ complex carrying RNA-binding proteins that regulate mRNA translation in response to developmental, physiological and environmental signals (39). We speculate that MERS-CoV nsp1 selectively targets nucleus-derived mRNAs, by binding to one of the mRNA-binding proteins that form the host mRNP complex, and inhibits the expression of host genes.

MERS-CoV nsp1 did not inhibit the translation of exogenous mRNAs, including MERS-CoV-like mRNA, that were introduced directly into the cytoplasm (Fig. 5). Furthermore, MERS-CoV nsp1 did not affect the translation and stability of a virus-like mRNA synthesized in the cytoplasm (Fig. 6). These data have important implications for the regulation of viral gene expression in MERS-CoV-infected cells and point toward a viral escape mechanism wherein MERS-CoV mRNAs, which are synthesized in the cytoplasm, are spared from the inhibitory effects of MERS- 
CoV nsp1 on mRNA translation. We hypothesize that the cytoplasmic origin of viral mRNAs facilitates their escape from MERS$\mathrm{CoV}$ nspl-induced translation inhibition allowing the efficient production of viral proteins. The reporter activity and the accumulation of $\mathrm{N}$ protein, from exogenous reporter mRNAs and MERS-CoV-like mRNA, respectively, that were introduced directly into the cytoplasm, was higher in cells expressing MERSCoV nsp1 but not in cells expressing MERS-CoV nsp1-CD, which lacked the RNA cleavage function (Fig. 5). We speculate that the degradation of endogenous host mRNAs by MERS-CoV nsp1 indirectly facilitates the translation of exogenously delivered mRNAs in the cytoplasm by eliminating the translationally competent host mRNAs that compete for the cellular translation machinery. However, it must be noted that this positive effect on the translation of exogenously introduced mRNAs by MERS-CoV nsp1 was not observed in the case of virus-like mRNAs that were synthesized in the cytoplasm (Fig. 6). This discrepancy could be due to differences in the experimental system and template mRNAs used to evaluate the effect of MERS-CoV nspl on the translation of mRNAs that originate in the cytoplasm. Nevertheless, this does not detract from our finding that cytoplasmically synthesized virus-like mRNAs are spared from the inhibitory effects of MERS-CoV nsp1. Future studies to examine the contribution of the host mRNA degradation activity of MERS-CoV nsp1 on viral mRNA translation in MERS-CoV-infected cells are warranted.

\section{ACKNOWLEDGMENTS}

We thank Heinz Feldmann and Ron A. Fouchier for providing the MERSCoV/EMC-2012 strain. We also thank Chikao Morimoto for pcDLSR $\alpha 296$, Britt A. Glaunsinger for the Pol I-, Pol II-, and Pol III-driven GFP reporter plasmids, and Adriana Paulucci-Holthauzen for support with confocal microscopy analysis.

This study was supported by Public Health Service grants AI99107, AI101772, AI114657, and AI117445 from the National Institutes of Health and a grant from the Institute for Human Infections and Immunity at The University of Texas Medical Branch. K. Nakagawa was supported by the James W. McLaughlin fellowship fund.

\section{REFERENCES}

1. Assiri A, McGeer A, Perl TM, Price CS, Al Rabeeah AA, Cummings DA, Alabdullatif ZN, Assad M, Almulhim A, Makhdoom H, Madani H, Alhakeem R, Al-Tawfiq JA, Cotten M, Watson SJ, Kellam P, Zumla AI, Memish ZA, Team KM-CI. 2013. Hospital outbreak of Middle East respiratory syndrome coronavirus. N Engl J Med 369:407-416. http://dx .doi.org/10.1056/NEJMoa1306742.

2. Memish ZA, Mishra N, Olival KJ, Fagbo SF, Kapoor V, Epstein JH, Alhakeem R, Durosinloun A, Al Asmari M, Islam A, Kapoor A, Briese T, Daszak P, Al Rabeeah AA, Lipkin WI. 2013. Middle East respiratory syndrome coronavirus in bats, Saudi Arabia. Emerg Infect Dis 19:18191823. http://dx.doi.org/10.3201/eid1911.131172.

3. Briese T, Mishra N, Jain K, Zalmout IS, Jabado OJ, Karesh WB, Daszak P, Mohammed OB, Alagaili AN, Lipkin WI. 2014. Middle East respiratory syndrome coronavirus quasispecies that include homologues of human isolates revealed through whole-genome analysis and virus cultured from dromedary camels in Saudi Arabia. mBio 5:e01146-14. http://dx.doi .org/10.1128/mBio.01146-14.

4. Azhar EI, El-Kafrawy SA, Farraj SA, Hassan AM, Al-Saeed MS, Hashem AM, Madani TA. 2014. Evidence for camel-to-human transmission of MERS coronavirus. N Engl J Med 370:2499-2505. http://dx.doi.org/10 .1056/NEJMoa1401505.

5. Memish ZA, Zumla AI, Al-Hakeem RF, Al-Rabeeah AA, Stephens GM. 2013. Family cluster of Middle East respiratory syndrome coronavirus infections. N Engl J Med 368:2487-2494. http://dx.doi.org/10.1056 /NEJMoa1303729.
6. Memish ZA, Zumla AI, Assiri A. 2013. Middle East respiratory syndrome coronavirus infections in health care workers. N Engl J Med 369:884-886. http://dx.doi.org/10.1056/NEJMc1308698.

7. Drosten C, Gunther S, Preiser W, van der Werf S, Brodt HR, Becker S, Rabenau H, Panning M, Kolesnikova L, Fouchier RA, Berger A, Burguiere AM, Cinatl J, Eickmann M, Escriou N, Grywna K, Kramme S, Manuguerra JC, Muller S, Rickerts V, Sturmer M, Vieth S, Klenk HD, Osterhaus AD, Schmitz H, Doerr HW. 2003. Identification of a novel coronavirus in patients with severe acute respiratory syndrome. N Engl J Med 348:1967-1976. http://dx.doi.org/10.1056/NEJMoa030747.

8. Guery B, Poissy J, el Mansouf L, Sejourne C, Ettahar N, Lemaire X, Vuotto F, Goffard A, Behillil S, Enouf V, Caro V, Mailles A, Che D, Manuguerra JC, Mathieu D, Fontanet A, van der Werf S, MERS-CoV study group. 2013. Clinical features and viral diagnosis of two cases of infection with Middle East respiratory syndrome coronavirus: a report of nosocomial transmission. Lancet 381:2265-2272. http://dx.doi.org/10 .1016/S0140-6736(13)60982-4.

9. Menachery VD, Eisfeld AJ, Schafer A, Josset L, Sims AC, Proll S, Fan S, Li C, Neumann G, Tilton SC, Chang J, Gralinski LE, Long C, Green R, Williams CM, Weiss J, Matzke MM, Webb-Robertson BJ, Schepmoes AA, Shukla AK, Metz TO, Smith RD, Waters KM, Katze MG, Kawaoka Y, Baric RS. 2014. Pathogenic influenza viruses and coronaviruses utilize similar and contrasting approaches to control interferon-stimulated gene responses. mBio 5:e01174-14. http://dx.doi.org/10.1128/mBio.01174-14.

10. Snijder EJ, Bredenbeek PJ, Dobbe JC, Thiel V, Ziebuhr J, Poon LL, Guan Y, Rozanov M, Spaan WJ, Gorbalenya AE. 2003. Unique and conserved features of genome and proteome of SARS-coronavirus, an early split-off from the coronavirus group 2 lineage. J Mol Biol 331:9911004. http://dx.doi.org/10.1016/S0022-2836(03)00865-9.

11. Prentice E, McAuliffe J, Lu X, Subbarao K, Denison MR. 2004. Identification and characterization of severe acute respiratory syndrome coronavirus replicase proteins. J Virol 78:9977-9986. http://dx.doi.org/10 $.1128 / J V I .78 .18 .9977-9986.2004$.

12. Newman BW, Chamberlain P, Bowden F, Joseph J. 2014. Atlas of coronavirus replicase structure. Virus Res 194:49-66. http://dx.doi.org /10.1016/j.virusres.2013.12.004.

13. Tohya Y, Narayanan K, Kamitani W, Huang C, Lokugamage K, Makino S. 2009. Suppression of host gene expression by nsp 1 proteins of group 2 bat coronaviruses. J Virol 83:5282-5288. http://dx.doi.org/10.1128/JVI .02485-08.

14. Kamitani W, Huang C, Narayanan K, Lokugamage KG, Makino S. 2009. A two-pronged strategy to suppress host protein synthesis by SARS coronavirus Nspl protein. Nat Struct Mol Biol 16:1134-1140. http://dx .doi.org/10.1038/nsmb.1680.

15. Kamitani W, Narayanan K, Huang C, Lokugamage K, Ikegami T, Ito N, Kubo H, Makino S. 2006. Severe acute respiratory syndrome coronavirus nsp1 protein suppresses host gene expression by promoting host mRNA degradation. Proc Natl Acad Sci U S A 103:12885-12890. http://dx.doi.org /10.1073/pnas.0603144103.

16. Huang C, Lokugamage KG, Rozovics JM, Narayanan K, Semler BL, Makino S. 2011. Alphacoronavirus transmissible gastroenteritis virus nsp1 protein suppresses protein translation in mammalian cells and in cell-free HeLa cell extracts but not in rabbit reticulocyte lysate. J Virol 85:638-643. http://dx.doi.org/10.1128/JVI.01806-10.

17. Zust R, Cervantes-Barragan L, Kuri T, Blakqori G, Weber F, Ludewig B, Thiel V. 2007. Coronavirus non-structural protein 1 is a major pathogenicity factor: implications for the rational design of coronavirus vaccines. PLoS Pathog 3:e109. http://dx.doi.org/10.1371/journal.ppat.0030109.

18. Tanaka T, Kamitani W, Dediego ML, Enjuanes L, Matsuura Y. 2012. Severe acute respiratory syndrome coronavirus nsp1 facilitates efficient propagation in cells through a specific translational shutoff of host mRNA. J Virol 86:11128-11137. http://dx.doi.org/10.1128/JVI.01700-12.

19. Lokugamage KG, Narayanan K, Huang C, Makino S. 2012. Severe acute respiratory syndrome coronavirus protein nsp1 is a novel eukaryotic translation inhibitor that represses multiple steps of translation initiation. J Virol 86:13598-13608. http://dx.doi.org/10.1128/JVI.01958-12.

20. Huang C, Lokugamage KG, Rozovics JM, Narayanan K, Semler BL, Makino S. 2011. SARS coronavirus nspl protein induces templatedependent endonucleolytic cleavage of mRNAs: viral mRNAs are resistant to nsp1-induced RNA cleavage. PLoS Pathog 7:e1002433. http://dx.doi .org/10.1371/journal.ppat.1002433.

21. Gaglia MM, Covarrubias S, Wong W, Glaunsinger BA. 2012. A common 
strategy for host RNA degradation by divergent viruses. J Virol 86:95279530. http://dx.doi.org/10.1128/JVI.01230-12.

22. Smiley JR. 2004. Herpes simplex virus virion host shutoff protein: immune evasion mediated by a viral RNase? J Virol 78:1063-1068. http://dx doi.org/10.1128/JVI.78.3.1063-1068.2004

23. Bouloy M, Janzen C, Vialat P, Khun H, Pavlovic J, Huerre M, Haller O. 2001. Genetic evidence for an interferon-antagonistic function of Rift Valley fever virus nonstructural protein NSs. J Virol 75:1371-1377. http://dx .doi.org/10.1128/JVI.75.3.1371-1377.2001.

24. Zhang R, Li Y, Cowley TJ, Steinbrenner AD, Phillips JM, Yount BL, Baric RS, Weiss SR. 2015. The nsp1, nsp13, and M proteins contribute to the hepatotropism of murine coronavirus JHM.WU. J Virol 89:3598 3609. http://dx.doi.org/10.1128/JVI.03535-14.

25. Zaki AM, van Boheemen S, Bestebroer TM, Osterhaus AD, Fouchier RA. 2012. Isolation of a novel coronavirus from a man with pneumonia in Saudi Arabia. N Engl J Med 367:1814-1820. http://dx.doi.org/10.1056 /NEJMoa1211721.

26. Murakami S, Terasaki K, Ramirez SI, Morrill JC, Makino S. 2014. Development of a novel, single-cycle replicable Rift Valley fever vaccine. PLoS Negl Trop Dis 8:e2746. http://dx.doi.org/10.1371/journal.pntd.0002746.

27. Taddeo B, Esclatine A, Roizman B. 2002. The patterns of accumulation of cellular RNAs in cells infected with a wild-type and a mutant herpes simplex virus 1 lacking the virion host shutoff gene. Proc Natl Acad Sci U S A 99:17031-17036. http://dx.doi.org/10.1073/pnas.252588599.

28. Rubio RM, Mora SI, Romero P, Arias CF, Lopez S. 2013. Rotavirus prevents the expression of host responses by blocking the nucleocytoplasmic transport of polyadenylated mRNAs. J Virol 87:6336-6345. http://dx .doi.org/10.1128/JVI.00361-13.

29. Murakami S, Terasaki K, Narayanan K, Makino S. 2012. Roles of the coding and noncoding regions of Rift Valley fever virus RNA genome segments in viral RNA packaging. J Virol 86:4034-4039. http://dx.doi.org /10.1128/JVI.06700-11.

30. Buchholz UJ, Finke S, Conzelmann KK. 1999. Generation of bovine respiratory syncytial virus (BRSV) from cDNA: BRSV NS2 is not essential for virus replication in tissue culture, and the human RSV leader region acts as a functional BRSV genome promoter. J Virol 73:251-259.

31. Raj VS, Mou H, Smits SL, Dekkers DH, Muller MA, Dijkman R, Muth
D, Demmers JA, Zaki A, Fouchier RA, Thiel V, Drosten C, Rottier PJ, Osterhaus AD, Bosch BJ, Haagmans BL. 2013. Dipeptidyl peptidase 4 is a functional receptor for the emerging human coronavirus-EMC. Nature 495:251-254. http://dx.doi.org/10.1038/nature12005.

32. Narayanan K, Huang C, Lokugamage K, Kamitani W, Ikegami T, Tseng CT, Makino S. 2008. Severe acute respiratory syndrome coronavirus nsp 1 suppresses host gene expression, including that of type I interferon, in infected cells. J Virol 82:4471-4479. http://dx.doi.org/10 .1128/JVI.02472-07.

33. Almeida MS, Johnson MA, Herrmann T, Geralt M, Wuthrich K. 2007. Novel beta-barrel fold in the nuclear magnetic resonance structure of the replicase nonstructural protein 1 from the severe acute respiratory syndrome coronavirus. J Virol 81:3151-3161. http://dx.doi.org/10.1128/JVI .01939-06.

34. Terasaki K, Makino S. 2015. Interplay between the virus and host in Rift Valley fever pathogenesis. J Innate Immun 7:450-458. http://dx.doi.org /10.1159/000373924.

35. Habjan M, Penski N, Wagner V, Spiegel M, Overby AK, Kochs G, Huiskonen JT, Weber F. 2009. Efficient production of Rift Valley fever virus-like particles: the antiviral protein MxA can inhibit primary transcription of bunyaviruses. Virology 385:400-408. http://dx.doi.org/10 $.1016 /$ j.virol.2008.12.011.

36. Narayanan K, Ramirez SI, Lokugamage KG, Makino S. 2015. Coronavirus nonstructural protein 1: common and distinct functions in the regulation of host and viral gene expression. Virus Res 202:89-100. http://dx .doi.org/10.1016/j.virusres.2014.11.019.

37. Tijms MA, van der Meer Y, Snijder EJ. 2002. Nuclear localization of nonstructural protein 1 and nucleocapsid protein of equine arteritis virus. J Gen Virol 83:795-800.

38. Yadani FZ, Kohl A, Prehaud C, Billecocq A, Bouloy M. 1999. The carboxy-terminal acidic domain of Rift Valley Fever virus NSs protein is essential for the formation of filamentous structures but not for the nuclear localization of the protein. J Virol 73:5018-5025.

39. Mitchell SF, Parker R. 2014. Principles and properties of eukaryotic mRNPs. Mol Cell 54:547-558. http://dx.doi.org/10.1016/j.molcel.2014 .04.033. 